\title{
Systematics of properties of the electron gas in deep-etched quantum wires
}

\author{
J. Martorell \\ Departamento d'Estructura i Constituents de la Materia, Facultat Física, University of Barcelona, Barcelona 08028, Spain \\ D. W. L. Sprung \\ Department of Physics and Astronomy, McMaster University, Hamilton, Ontario, Canada L8S 4M1
}

(Received 2 June 1995; revised manuscript received 24 May 1996)

\begin{abstract}
An efficient method is developed for an iterative solution of the Poisson and Schrödinger equations, which allows systematic studies of the properties of the electron gas in linear deep-etched quantum wires. A much simpler two-dimensional (2D) approximation is developed that accurately reproduces the results of the 3D calculations. A 2D Thomas-Fermi approximation is then derived, and shown to give a good account of average properties. Further, we prove that an analytic form due to Shikin et al. is a good approximation to the electron density given by the self-consistent methods. [S0163-1829(96)00339-6]
\end{abstract}

\section{INTRODUCTION}

Growing interest in the development of devices based on $\mathrm{GaAs} / \mathrm{Al}_{x} \mathrm{Ga}_{1-x} \mathrm{As}$ deep-etched wires in nanoelectronics and related fields has lead to several detailed attempts ${ }^{1,2}$ to model the properties of the quasi-two-dimensional electron gas (2DEG) that appears under suitable design conditions in such wires. In general, these attempts focus on the detailed description of a specific device for which experimental measurements are available, and less effort is put into identifying general properties of the $2 \mathrm{DEG}$ in deep-etched wires or in devising fast numerical methods applicable to different general situations. In Sec. II, we shall present methods to solve the coupled Poisson and Schrödinger equations that allow significant improvement in the speed of numerical calculations and that furthermore can be applied under rather general conditions.

The main objective of the present work, however, is to get a more transparent understanding of the connection between the geometry of the deep-etched wire, the physical properties of $\mathrm{GaAs}$ and $\mathrm{Al}_{x} \mathrm{Ga}_{1-x} \mathrm{As}$, and the properties of the distribution of charge in the 2DEG. With this purpose, and using as a reference the results of the complete PoissonSchrödinger calculations, we will derive successive approximations which reveal this connection. The first of these is presented in Sec. IV: it is a factorization ansatz based on analytic expressions for the confining potential derived in Sec. III. This ansatz allows an accurate separation of the longitudinal (vertical according to Fig. 1) and transverse components of the wave functions of the electrons in the gas. This provides a quantitative foundation for those more phenomenological models that assume from the beginning that the electron gas is purely two dimensional (2D). Based on this separation, in Sec. V we formulate a 2D Thomas-Fermi approximation and verify that it also predicts with good accuracy the bulk properties of the electron distribution in the gas. Finally, in Sec. VI, we make the connection with a purely classical model proposed by Shikin et $a l^{3,4}$ for the charge distribution. We show that the model can be applied to our description and leads to a set of analytic relations, Eqs. (53), (54), and (55) that directly relate the parameters defin- ing the electron charge distribution to the geometry and physical properties of the heterostructure.

In summary, by developing successive approximations we make the connection between the complete, but cumbersome, 3D Poisson-Schrödinger calculations, and simpler models of the 2DEG in deep-etched wires. We show that although the latter cannot reproduce the fine details of the fully quantal calculations, they provide a physically intuitive picture of the results of the numerical calculations and a reasonably accurate prediction of the bulk properties via analytic expressions.

\section{PROPERTIES OF THE THREE-DIMENSIONAL ELECTRON GAS}

Although the methods discussed below are of general utility, we consider a specific deep-etched wire for definiteness. Figure 1 shows this heterojunction configuration, similar to the deep mesa structure already studied in Ref. 1. From top to bottom we take $15,20,4,16$, and $10 \mathrm{~nm}$ for the thicknesses of the layers shown, and we write $w$ for the width. The cap and donor layers are $n$ doped, with $\rho_{d}=10^{18} \mathrm{~cm}^{-3}$, and we assume that the band offset between the two materials is $e V_{b}=0.23 \mathrm{eV}$. For simplicity we neglect the difference in their permittivities and use a common relative permittivity, $\varepsilon_{r}=13.1$.

In our model we assume that, due to the etching process, surface states form in the exposed parts of the wire, so as to induce Fermi level pinning. This has been verified in a number of experimental conditions. ${ }^{5}$ We further assume that the Fermi level on the surface of the heterojunction is the same everywhere, and choose it as the zero of energies. Accordingly, we set the location of the conduction band at points on the surface of GaAs at a fixed energy $e V_{s}$ and on the surface of $\mathrm{Al}_{x} \mathrm{Ga}_{1-x} \mathrm{As}$ at $e V_{s}+e V_{b}$. Although there is no clear experimental evidence for the validity of these assumptions, they are certainly compatible with recent work supporting the interpretation of surface states as localized defects whose ground state wave function is of a bonding type, and with the variation with composition of the energy of these states in $n-\mathrm{Al}_{x} \mathrm{Ga}_{1-x} \mathrm{As}$ samples. ${ }^{6}$ For ease of comparison with pre- 


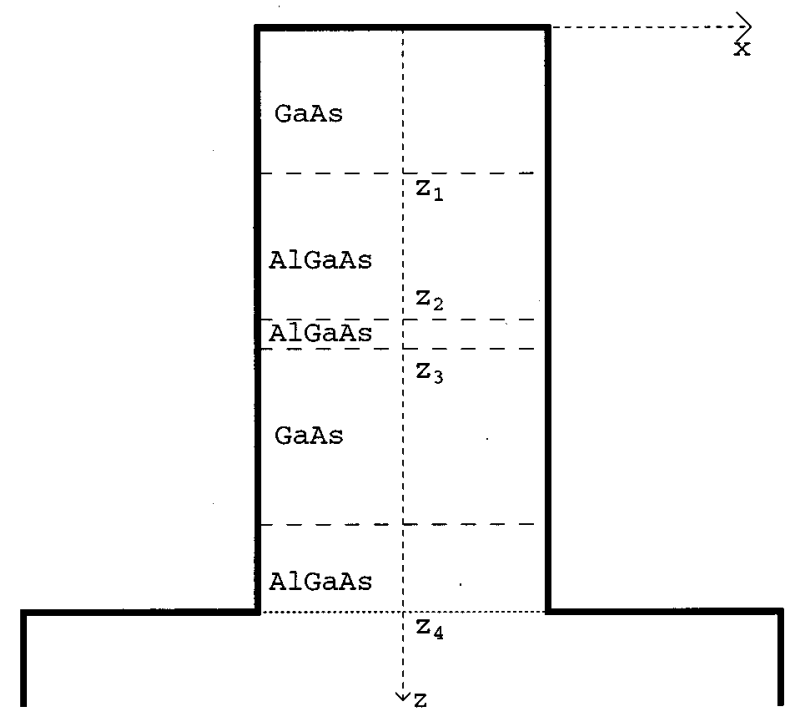

FIG. 1. Section of the deep-etched heterostructure.

vious simulations we have chosen $e V_{s}=0.6 \mathrm{eV}$.

The electrostatic potential due to the charges present in the heterostructure is different at each point, and therefore its variation affects the energy, $e \Phi$, of the bottom of the conduction band inside the material. Since the etched wire is translationally invariant along the $y$ axis, we write this energy as the sum

$$
e \Phi(x, z)=e V_{s}+e V_{b}(z)+e V(x, z),
$$

where the contribution due to the band offset, $e V_{b}(z)$, is zero in the GaAs layers and $0.23 \mathrm{eV}$ in the $\mathrm{Al}_{x} \mathrm{Ga}_{1-x}$ As layers. The term due to the electrostatic potential, $\mathrm{eV}(x, z)$, is determined by solving the Poisson equation with the boundary condition that it vanishes on all the exposed surfaces (shown as thick horizontal and vertical lines in Fig. 1). This boundary condition follows from our choice of the Fermi level as the origin of energies and the use of Eq. (1) to define the bottom of the conduction band. We write the Poisson equation as

$$
e \Delta V(x, z)=\frac{e^{2}}{\varepsilon} \rho(x, z),
$$

where in the zone of the ionized cap and donor layers $\rho(x, z)=\rho_{d}=$ const $>0$, whereas in the zone of the 2DEG, $\rho(x, z)=-\rho_{e}(x, z)<0$. When fixed value boundary conditions are required on two parallel sides of a rectangle, as here, this equation can be very efficiently solved using Fourier series expansions. We therefore write expansions for the potential and the density as

$$
\begin{gathered}
e V(x, z)=\sum_{n=0}^{\infty} v_{n}(z) \cos \beta_{n} x, \\
\rho(x, z)=\sum_{n=0}^{\infty} \rho_{n}(z) \cos \beta_{n} x,
\end{gathered}
$$

where $\beta_{n} \equiv(2 n+1) \pi / w$. Orthogonality of the $\cos \left(\beta_{n} x\right)$ leads to

$$
v_{n}^{\prime \prime}(z)-\beta_{n}^{2} v_{n}(z)=\frac{e^{2}}{\varepsilon} \rho_{n}(z),
$$

with

$$
\rho_{n}(z) \equiv \frac{2}{w} \int_{-w / 2}^{w / 2} \rho(x, z) \cos \left(\beta_{n} x\right) d x .
$$

The explicit form of the solution, $v_{n}(z)$, is different in each zone of the heterostructure. It is convenient to construct solutions for each zone separately and then match them at the intervening horizontal boundaries. The general form of the $v_{n}(z)$ in the $i$ th zone that is well suited for this process is

$$
\begin{gathered}
v_{n}(z)=v_{n}^{(0)}(z)+v_{n}^{(c h)}(z), \\
v_{n}^{(0)}(z)=C_{n,+} e^{\beta_{n}\left(z-z_{i}\right)}+C_{n,-} e^{-\beta_{n}\left(z-z_{i}\right)}, \\
v_{n}^{(c h)}(z)=\frac{e^{2}}{\varepsilon \beta_{n}} \int_{z_{i}}^{z} \sinh \beta_{n}\left(z-z^{\prime}\right) \rho_{n}\left(z^{\prime}\right) d z^{\prime} .
\end{gathered}
$$

We are now going to particularize these results to each separate region and impose the appropriate matching conditions.

Zone 1. $\left(0 \leqslant z \leqslant z_{2}\right)$. It is convenient to start with the topmost zone because the boundary condition at $z=0$ is simply $e V(x, z=0)=0$. Here, $\rho(x, z)=\rho_{d}=$ const and therefore

$$
\rho_{n}(z)=\frac{(-1)^{n}}{2 n+1} \frac{4 \rho_{d}}{\pi},
$$

so by requiring $v_{n}(0)=0$ one finds

$$
v_{n}(z)=\frac{e^{2} \rho_{d} w^{2}}{\varepsilon}\left[a_{n} \sinh \beta_{n} z+\gamma_{n}\left(\cosh \beta_{n} z-1\right)\right],
$$

where $\gamma_{n} \equiv 4(-1)^{n} /[(2 n+1) \pi]^{3}$ and the $a_{n}$ will be determined by matching to the next zone.

Zone 2. $\left(z_{2} \leqslant z \leqslant z_{4}\right)$. This corresponds to the spacer layer and that part of the substrate layer in the etched wire. Here, the $\rho_{n}(z)$ are determined by substituting in Eq. (5) the (yet to be determined) electron gas density. Imposing the matching conditions,

$$
\begin{aligned}
& v_{n}\left(z_{2}-0\right)=v_{n}\left(z_{2}+0\right), \\
& v_{n}^{\prime}\left(z_{2}-0\right)=v_{n}^{\prime}\left(z_{2}+0\right),
\end{aligned}
$$

one finds

$$
\begin{aligned}
v_{n}(z)= & \frac{e^{2} \rho_{d} w^{2}}{\varepsilon} \\
& \times\left\{a_{n} \sinh \beta_{n} z+\gamma_{n}\left[\cosh \beta_{n} z-\cosh \beta_{n}\left(z-z_{2}\right)\right]\right\} \\
& +v_{n}^{(c h)}(z) .
\end{aligned}
$$

Zone 3. $\left(z \geqslant z_{4}\right)$ corresponds to the substrate below the mesa. We will denote its width by $W$ (which in the numerical calculations is chosen sufficiently large so that the results are equivalent to letting $W \rightarrow \infty$ ). For the deep-etched wires that we are studying here, it is a good approximation to neglect 
the electron density in this zone. We therefore introduce a set of solutions of the Laplace equation with boundary conditions chosen so as to make them an appropriate basis for expanding $e V(x, z)$. These solutions, $\Lambda_{m}(x, z)$, are of the form

$$
\Lambda_{m}(x, z)=\sum_{i=0}^{\infty} \lambda_{i, m} e^{-\alpha_{i}\left(z-z_{4}\right)} \cos \alpha_{i} x,
$$

with $\alpha_{i}=(2 i+1) \pi / W$. And imposing them to satisfy

$$
\begin{gathered}
\Lambda_{m}\left(x= \pm W / 2, z>z_{4}\right)=0, \\
\Lambda_{m}\left(x, z=z_{4}\right)=\cos \beta_{m} x \Theta\left(\frac{w}{2}-|x|\right), \\
\Lambda_{m}(x, z \rightarrow \infty)=0,
\end{gathered}
$$

$[\Theta(x)$ is the Heaviside step function], leads to

$$
\begin{aligned}
\lambda_{i, m}= & \frac{w}{W}\left[j_{0}\left(\left(\alpha_{i}+\beta_{m}\right) \frac{w}{2}\right)+j_{0}\left(\left(\alpha_{i}-\beta_{m}\right) \frac{w}{2}\right)\right] \\
= & \zeta\left(j_{0}\left\{\left[\left(i+\frac{1}{2}\right) \zeta+m+\frac{1}{2}\right] \pi\right\}\right. \\
& \left.+j_{0}\left\{\left[\left(i+\frac{1}{2}\right) \zeta-m-\frac{1}{2}\right] \pi\right\}\right),
\end{aligned}
$$

where $\zeta \equiv w / W$. The matching condition on $e V(x, z)$ at $z=z_{4}$ then allows one to write

$$
e V(x, z)=\sum_{m=0}^{\infty} v_{m}\left(z_{4}-0\right) \Lambda_{m}(x, z),
$$

and requiring that the $\partial e V(x, z) / \partial z$ also match at $z_{4}$, we obtain the remaining condition needed to determine completely the solution of the Poisson equation:

$$
v_{n}^{\prime}\left(z_{4}-0\right)=-\frac{W}{w} \sum_{m=0}^{\infty} v_{m}\left(z_{4}-0\right) \epsilon_{m, n},
$$

with

$$
\epsilon_{m, n} \equiv \sum_{i=0}^{\infty} \alpha_{i} \lambda_{i, m} \lambda_{i, n}
$$

This leads to a system of coupled linear equations for the unknown coefficients $a_{m}, m=0,1, \ldots$ of the form

$$
\sum_{m} B_{n, m} a_{m}=D_{n}
$$

with

$$
B_{n, m} \equiv \frac{e^{2} \rho_{d} w^{2}}{\varepsilon}\left(-\frac{W}{w} \epsilon_{m, n} \sinh \beta_{m} z_{4}-\beta_{n} \delta_{n, m} \cosh \beta_{n} z_{4}\right)
$$

and

$$
\begin{aligned}
D_{n} \equiv & \frac{W}{w} \sum_{m} \epsilon_{m, n}\left[\frac{e^{2} \rho_{d} w^{2}}{\varepsilon} \gamma_{m}\left[\cosh \beta_{m} z_{4}-\cosh \beta_{m}\left(z_{4}-z_{2}\right)\right]\right. \\
& \left.+v_{m}^{(c h)}\left(z_{4}\right)\right]+\frac{e^{2} \rho_{d} w^{2}}{\varepsilon} \beta_{n} \gamma_{n}\left[\sinh \beta_{n} z_{4}\right.
\end{aligned}
$$

$$
\left.-\sinh \beta_{n}\left(z_{4}-z_{2}\right)\right]+v_{n}^{\prime(\mathrm{ch})}\left(z_{4}\right) .
$$

After solving Eq. (17) for the $a_{m}$, the integration of Poisson's equation is completed by substituting these values in Eqs. (8) and (10) to determine the $v_{n}(z)$ in the zones of interest and then using Eq. (3) to construct the potential. Details of the numerical solution of Eq. (17) are given in Appendix A and in the next section.

Determination of the subband wave functions. In the envelope function approximation the wave functions of the electrons in the 2DEG are determined by solving the Schrödinger equation with potential $e \Phi(x, z)$, as defined in Eq. (1). Due to invariance along the $y$ axis they take the factorized form

$$
\Psi_{l, k_{y}}(x, y, z)=\frac{1}{\sqrt{2 \pi}} e^{i k_{y} y} \Psi_{l}(x, z),
$$

where the $\Psi_{l}(x, z)$ satisfy

$$
\left[-\frac{\hbar^{2}}{2 m^{*}}\left(\frac{d^{2}}{d x^{2}}+\frac{d^{2}}{d z^{2}}\right)+e \Phi(x, z)\right] \Psi_{l}(x, z)=E_{l} \Psi_{l}(x, z) .
$$

We have solved this two-dimensional equation using the same numerical methods described in Ref. 7: we define a rectangular mesh of points in the spacer and substrate and discretize the differential equation reducing it to a matrix eigenvalue problem. Then a Lanczos algorithm is used to find the lowest eigenvalues and eigenfunctions, which are the only ones needed here. When required, we improve the accuracy by performing inverse iteration, as proposed in Sec. 11.7 of Ref. 8. These are the more time consuming parts of the numerical calculations. Even so, the typical running times are 2 min per iteration on a 486DX-33 PC, a considerable improvement over those quoted by Snider et al. in Ref. 1.

Due to the assumption of thermal equilibrium, we write the subband occupations as

$$
f_{l}=\frac{1}{\pi} \sqrt{\frac{2 m^{*} k_{B} T}{\hbar^{2}}} F_{-1 / 2}\left(\frac{E_{F}-E_{l}}{k_{B} T}\right),
$$

where $F_{-1 / 2}$ is the Fermi-Dirac integral, and we recall that our origin of energies sets $E_{F}=0$. We then construct the electron densities from

$$
\rho_{e}(x, z)=\sum_{l=1}^{L} f_{l}\left|\Psi_{l}(x, z)\right|^{2},
$$

with the sum truncated when the $f_{l}$ become negligible. The two-dimensional and the linear densities are

$$
\begin{gathered}
\sigma(x)=\int_{z_{2}}^{\infty} \rho_{e}(x, z) d z, \\
\lambda=\int_{-\infty}^{\infty} \sigma(x) d x .
\end{gathered}
$$

By iteratively solving the Poisson and Schrödinger equations, a self-consistent solution for all these quantities is determined for a given width $w$.

For brevity we present results only for the $T=0 \mathrm{~K}$ case. (The systematics of the results at finite temperature are quali- 


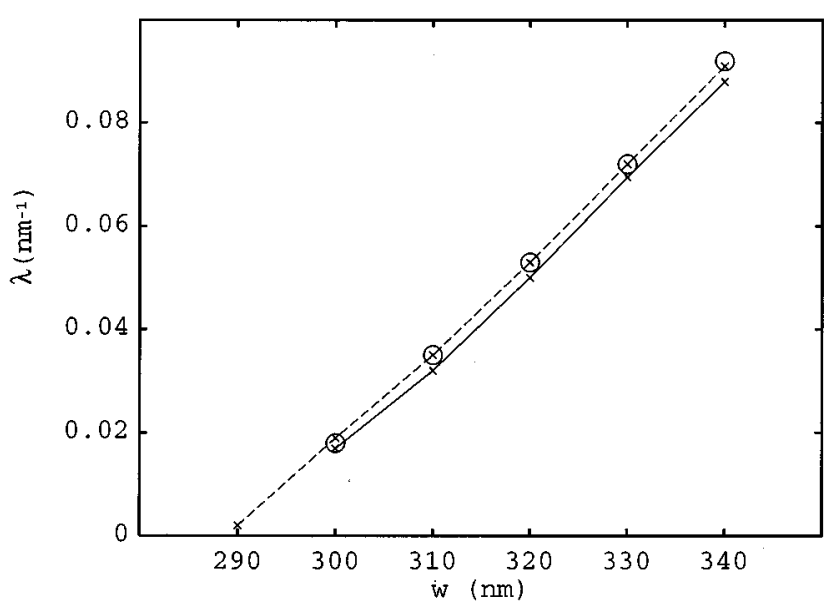

FIG. 2. Variation of linear density with wire width. Continuous line: Poisson-Schrödinger calculation. Dashed line: 2D approximation. Circles: Thomas-Fermi predictions.

tatively similar to those found in Ref. 7 for a linear split gate wire.) The variation with wire width of the linear and twodimensional densities is shown in Figs. 2 and 3, respectively. It can be seen that there is a rather smooth and almost linear increase of $\lambda$ with the width, $w$. The two-dimensional density also increases both in width and central value with $w$, and shows oscillations due to the changes in the filling of each subband. We find that $\sigma(x)$ becomes rather smooth as more subbands are filled when the width increases. As expected, calculations at finite $T$ lead to even smoother profiles. The longitudinal confining potential at $x=0, e \Phi(0, z)$ is plotted in Fig. 4 together with $\rho_{e}(0, z)$; clearly the band offsets are a determinant in the confinement of the electron gas, and the quasilinear increase of the potential in the GaAs substrate is responsible for the slight asymmetry in the profile of the electron density. The quantitative correlation between changes in the densities and those of the confining potential will be discussed in detail in the following sections.

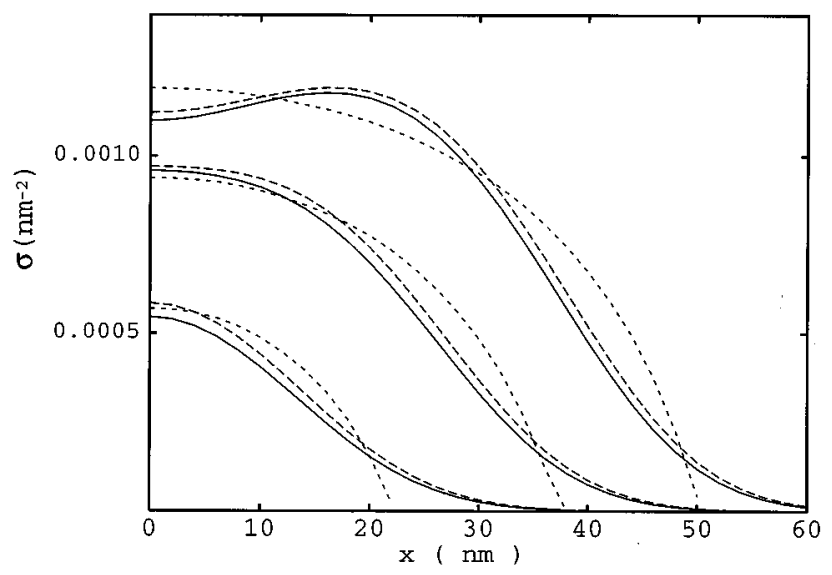

FIG. 3. Two-dimensional densities for wire widths: $w=300$, 320 , and $340 \mathrm{~nm}$. Continuous line: Poisson-Schrödinger calculation. Long dashed line: 2D approximation. Dotted line: ThomasFermi prediction.

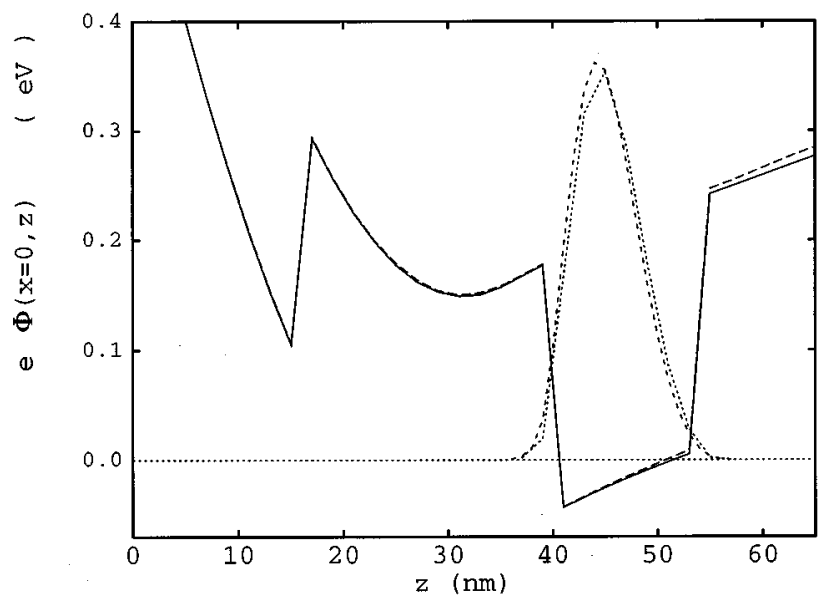

FIG. 4. Longitudinal confining potential for wire widths $w=320 \mathrm{~nm}$ (continuous line) and $300 \mathrm{~nm}$ (long dashed line.) The 2DEG densities for $w=320 \mathrm{~nm}$ are also shown on an arbitrary scale. Dotted line: Poisson + Schrödinger result. Dashed line: 2D approximation.

\section{ANALYTICAL APPROXIMATIONS}

In the previous section, our implementation of the Poisson-Schrödinger approach lead to a satisfactory numerical description of the 2DEG properties. However, the interrelation between geometrical properties of the device, donor concentration, etc., and the characteristics of the electron gas is obscured by the complexity of the numerical computation. In the following sections we introduce approximations that without serious loss of accuracy provide a much more direct connection between the "input parameters" of the model and the final results for the 2DEG.

(1) Infinitely deep etched wire. We begin by studying this extreme limiting case, because it turns out to be a useful starting point for more elaborate approximations. We define such an ideal wire as one for which the width is the same for all values of $z$. We therefore set $W=w$ in the previous expressions, and the following simplifications apply:

$$
\begin{gathered}
\alpha_{i}^{(\infty)}=\beta_{i}, \\
\lambda_{i, m}^{(\infty)}=\delta_{i, m}, \\
\epsilon_{m, n}^{(\infty)}=\beta_{n} \delta_{m, n}, \\
B_{n, m}^{(\infty)}=-\frac{e^{2} \rho_{d} w^{2}}{\varepsilon} \beta_{n} e^{\beta_{n} z_{4}} \delta_{n, m}, \\
D_{n}^{(\infty)}=-\frac{e^{2} \rho_{d} w^{2}}{\varepsilon} \gamma_{n} \beta_{n} e^{\beta_{n} z_{4}}\left(e^{\left.-\beta_{n} z_{2}-1\right)}\right. \\
+\frac{e^{2}}{\varepsilon} \int_{z_{2}}^{z_{4}} e^{\beta_{n}\left(z_{4}-z\right)} \rho_{n}(z) d z,
\end{gathered}
$$

where $\delta_{n, m}$ is the Kronecker symbol. Since $B$ in Eq. (17) is now diagonal the solution for the $a_{n}$ is immediate, 


$$
a_{n}^{(\infty)}=\gamma_{n}\left(e^{\left.-\beta_{n} z_{2}-1\right)}-\frac{1}{\rho_{d} w^{2} \beta_{n}} \int_{z_{2}}^{z_{4}} e^{-\beta_{n} z} \rho_{n}(z) d z,\right.
$$

and therefore, for Zone 2 one finds

$$
\begin{aligned}
v_{n}^{(\infty)}(z)= & \frac{e^{2} \rho_{d} w^{2}}{\varepsilon} \gamma_{n}\left(1-\cosh \beta_{n} z_{2}\right) e^{-\beta_{n} z}+v_{n}^{(c h)}(z) \\
& -\frac{e^{2}}{\varepsilon \beta_{n}} e^{-\beta_{n} z_{2}} \int_{z_{2}}^{z_{4}} e^{-\beta_{n}\left(z^{\prime}-z_{2}\right)} \rho_{n}\left(z^{\prime}\right) d z^{\prime} \sinh \beta_{n} z
\end{aligned}
$$

The first term on the right hand side (r.h.s.) was already derived by Davies ${ }^{9}$ in the limit of vanishing electron gas density (pinch-off.) Indeed, the remaining terms account for the effect of the electrons in the gas and their contribution is comparatively small for the widths considered here.

(2) Wide base approximation (wba). To motivate this alternative approximation we note that, since we are interested in the limit $W \gg w$, it is natural to look for approximations where the sum over $i$ in Eq. (16) is replaced by an integral over a suitably defined continuous variable. The $\lambda_{i, m}$ defined in Eq. (13) satisfy an orthogonality relation:

$$
\sum_{i=0}^{\infty} \lambda_{i, m} \lambda_{i, n}=\frac{w}{W} \delta_{n, m} \equiv \zeta \delta_{n, m}
$$

which should be preserved. With this in mind one easily checks that by introducing

$$
\lambda_{m}(t)=\zeta\left\{j_{0}\left[\left(\zeta t+m+\frac{1}{2}\right) \pi\right]+j_{0}\left[\left(\zeta t-m-\frac{1}{2}\right) \pi\right]\right\}
$$

and then replacing the sum over $i$ by an integration from $t=0$ to $\infty$, the corresponding orthogonality relation still holds:

$$
\int_{0}^{\infty} d t \lambda_{m}(t) \lambda_{n}(t)=\zeta j_{0}[(m-n) \pi]=\zeta \delta_{n, m} .
$$

Applying the same prescription to Eq. (16) and with some minor simplifications in the integrals for the small terms, we obtain

$$
\begin{aligned}
\boldsymbol{\epsilon}_{m, n}^{(\mathrm{wba})}= & (2 n+1) \frac{\pi}{W} \delta_{m, n} \\
& +\frac{(-1)^{n+m}}{\pi W} \frac{(2 n+1)(2 m+1)}{(n+m+1)(n-m)} \ln \frac{2 n+1}{2 m+1} .
\end{aligned}
$$

The values predicted with this expression are in good quantitative agreement with the exact matrix elements computed numerically, the largest differences being of $\simeq 10 \%$ for the case $n=m=1$, and $\simeq 5 \%$ when $n=1, m=2$ and they quickly become negligible with increasing $n$ or $m$. The first term in the r.h.s. of Eq. (30) is always much larger than the second, and therefore the diagonal terms are always orders of magnitude larger than the nondiagonal. We have found however that due to large cancellations between the bigger terms, the nondiagonal matrix elements are needed to get accurate results. For a better qualitative understanding it is nevertheless interesting to derive the expressions that correspond to retaining only the first term on the r.h.s. of Eq. (30):

$$
\epsilon_{m, n}^{(\mathrm{wba}, 0)}=(2 n+1) \frac{\pi}{W} \delta_{m, n} .
$$

Placing this in Eq. (18), we find surprisingly that

$$
\begin{aligned}
& B_{n, m}^{(\mathrm{wba}, 0)}=B_{n, m}^{(\infty)}, \\
& D_{n}^{(\mathrm{wba}, 0)}=D_{n}^{(\infty)},
\end{aligned}
$$

and thus that the $a_{n}$ and $v_{n}$ are identical to those for the infinitely deep-etched wire given above. Since they emerge in these two very different limits, they should be a reasonable first approximation for understanding the main trends of the numerical calculations of Sec. II. Further, as we show in Appendix A, these expressions provide the starting point for an efficient numerical solution of Eq. (17) in the realistic case discussed in the previous section.

(3) Qualitative features. The first term in Eq. (26) for $v_{n}(z)$ is independent of the electron gas density. Its contribution to the electrostatic potential will be denoted by $e V_{0}$. Substituting into Eq. (3), one has

$e V_{0}(x, z)=-\frac{e^{2} \rho_{d} w^{2}}{\varepsilon} \operatorname{Re}\left[\sum_{n=0}^{\infty} \gamma_{n}\left(\cosh \beta_{n} z_{2}-1\right) e^{-\beta_{n} z} e^{i \beta_{n} x}\right]$

For simplicity we first discuss the case $x=0$. (Replacing $z$ by $z-i x$ and taking the real part, one obtains immediately the results for the $x \neq 0$ case.)

$$
\begin{aligned}
e V_{0}(0, z)= & -\frac{e^{2} \rho_{d} w^{2}}{\varepsilon} \sum_{n=0}^{\infty} \gamma_{n}\left(\cosh \beta_{n} z_{2}-1\right) e^{-\beta_{n} z} \\
= & -\frac{2 e^{2} \rho_{d} w^{2}}{\pi^{3} \varepsilon}\left[S\left(\xi=e^{-\nu\left(z-z_{2}\right)}\right)\right. \\
& \left.+S\left(\xi=e^{-\nu\left(z+z_{2}\right)}\right)-2 S\left(\xi=e^{-\nu z}\right)\right],
\end{aligned}
$$

where $\nu=\pi / w$ and we have defined

$$
S(\xi)=\sum_{n=0}^{\infty} \frac{(-1)^{n}}{(2 n+1)^{3}} \xi^{2 n+1} .
$$

Since the arguments in the exponentials in Eq. (34) are small when $z$ corresponds to the zone occupied by the 2DEG, one can obtain a very accurate estimate for $e V_{0}(0, z)$ by expanding $S[\xi=\exp (-\varepsilon)]$ in a Taylor series around $\varepsilon=0$ (and thus $\xi=1)$. In Appendix B, we show that

$$
\begin{aligned}
S\left(\xi=e^{-\varepsilon}\right)= & S(1)-\varepsilon S^{\prime}(1)+\frac{\pi}{8} \varepsilon^{2}-\frac{1}{12} \varepsilon^{3}+\frac{1}{240} \varepsilon^{5} \\
& -\frac{1}{2016} \varepsilon^{7}+O\left(\varepsilon^{9}\right),
\end{aligned}
$$

and the corresponding expansion for Eq. (34) becomes

$$
e V_{0}(0, z)=-\frac{e^{2} \rho_{d} z_{2}^{2}}{\varepsilon}\left(\frac{1}{2}-\frac{z}{w}+\frac{\pi^{2}}{12} \frac{z z_{2}^{2}+2 z^{3}}{w^{3}}\right) \text {. }
$$

From this result we determine the pinch-off width in the infinite etching limit: place the approximation Eq. (37) in Eq. (1) and set $e \Phi\left(0, z_{3}+0\right)=0$; then solving for $w$ gives 

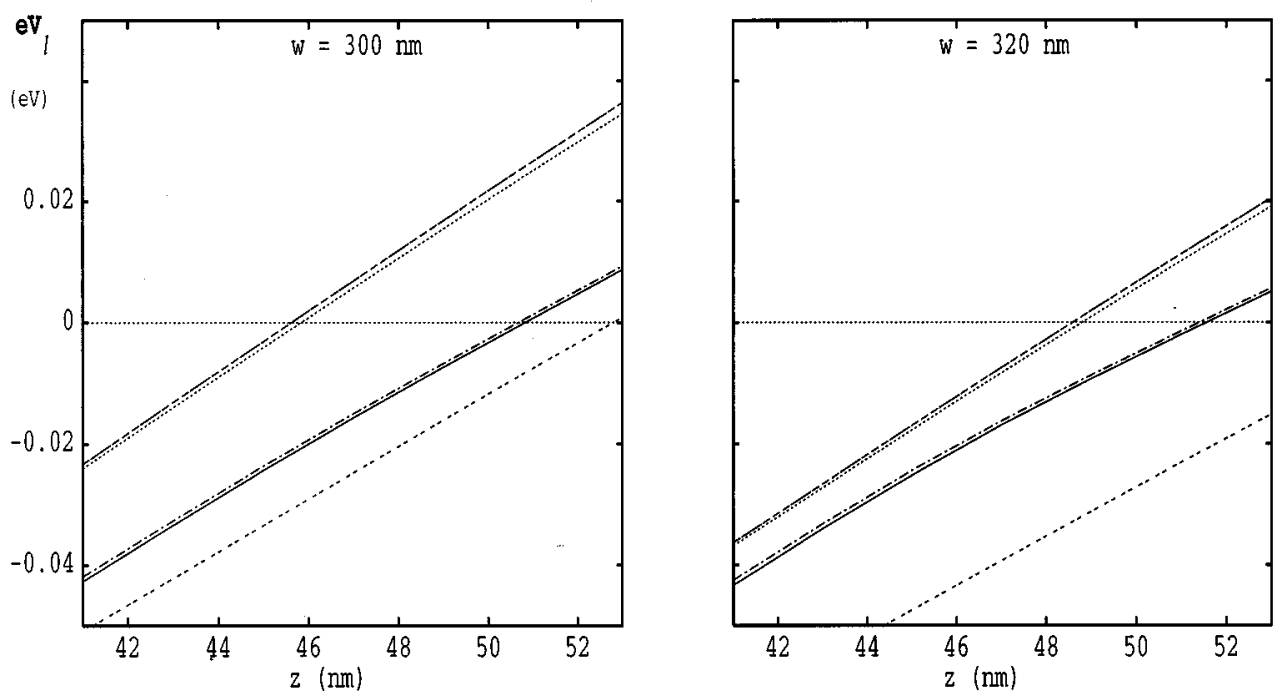

FIG. 5. Longitudinal confining potential in the substrate occupied by the electron gas, for two wire widths. Continuous line: $e \Phi(0, z)$ from the Poisson-Schrödinger calculation. Dot-dashed line: same, from the 2D approximation. Doubly dashed line: solution of the Poisson equation without electron gas, fulfilling the same boundary conditions. (Top) Dashed line: $e \Phi_{0}(0, z)$ obtained from the sum of the Fourier series in Eq. (34). Dotted line: same, obtained now from the analytic approximation in Eq. (37).

$$
w_{\mathrm{po}}=\frac{2 z_{3}}{1-\frac{2 e V_{s} \varepsilon}{e^{2} \rho_{d} z_{2}^{2}}}\left(1-\frac{\pi^{2}}{12} \frac{z_{2}^{2}+2 z_{3}^{2}}{w^{2}} \ldots\right) .
$$

Neglecting the term proportional to $\pi^{2}$ gives an accuracy of a few percent, which is improved if we iterate the value of $w$ in the right hand side.

To study in more detail the variation with $z$ in the zone of the 2DEG, in Fig. 5 we compare $e \Phi(0, z)$ to

$$
e \Phi_{0}(0, z) \equiv e V_{s}+e V_{0}(0, z)+e V_{b}(z)
$$

for two values of the width. First of all, it should be noted that the analytic result Eq. (37) is almost indistinguishable on this scale from the exact calculation based on Eq. (33). The

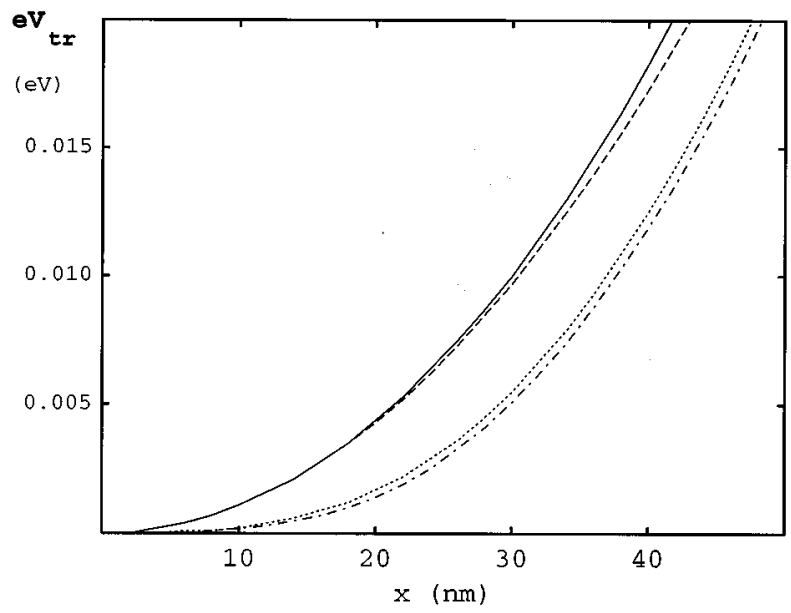

FIG. 6. Transverse confining potential for $w=320 \mathrm{~nm}$ and $z=43 \mathrm{~nm}$. Continuous line: $e V_{0}(x, z)-e V_{0}(0, z)$ from summing the Fourier series; long dashed line: quadratic approximation. Dotted line $e V(x, z)-e V(0, z)$ from the Poisson-Schrödinger solution; dash-dotted line: $2 \mathrm{D}$ approximation: $U(x)-U(0)$. difference between these and the Poisson-Schrödinger result, $e \Phi(0, z)$, is due to two effects: (i) the presence of the 2DEG, and (ii) the finite depth of the etching. To separate their contributions, we have set $\rho_{e}(x, z)=0$ and solved again Eq. (2) with the same boundary condition. This gives the doubly dashed line in Fig. 5. The difference between that line and $e \Phi(0, z)$ then accounts for (i), whereas the difference with $e \Phi_{0}(0, z)$ accounts for (ii). For a qualitative understanding of (ii) one can argue that the effect of an infinite etching of the wire amounts to bringing the lateral $z>z_{4}$ boundaries closer to the zone of the 2DEG. Since in these the energy of the conduction band is maximal: $e \Phi$ (boundary) $=e V_{s}+e V_{b}$, this induces a rise of the bottom of the conduction band in the zone of the 2 DEG by $\simeq 30 \mathrm{meV}$.

Note, in addition, that for each of the two widths shown, the slope corresponding to the quasilinear dependence on $z$ is little affected by (i) and (ii) and therefore can be well determined with the approximate expression Eq. (37). That expression also shows that when the width increases, both the position of the conduction band, $e \Phi_{0}(0, z)$, and its slope must decrease, which is indeed what is seen by comparing the plots for $w=300,320 \mathrm{~nm}$. This lowering of the conduction band is, however, compensated by the increased repulsion due to (i) because the electron gas density increases with $w$. The net result is then a rough independence of $e \Phi(0, z)$ on the value of $w$.

The same analytic expansion can be carried out for points $(x, z)$ not too far from the $z$ axis: it suffices to add an imaginary part $-i x$ to the $\varepsilon$ in Eq. (36). The lowest order result is

$$
e V_{0}(x, z)=e V_{0}(0, z)+\frac{e^{2} \rho_{d} z_{2}^{2} \pi^{2}}{2 \varepsilon w^{3}} z x^{2}
$$

In Fig. 6 we compare the exact $e \delta V_{0} \equiv e V_{0}(x, z)$ $-e V_{0}(0, z)$ to this quadratic approximation for a wire of width $w=300 \mathrm{~nm}$ and a value $z=43 \mathrm{~nm}$ that corresponds to the maximum density for the $2 \mathrm{DEG}$. Indeed to a very good 
approximation $e \delta V_{0}$ has the parabolic dependence on $x$ predicted by Eq. (40) in the region occupied by the 2DEG. In the same figure we also plot $e \delta V \equiv e V(x, z)-e V(0, z)$ [and $\delta U \equiv U(x)-U(0)$, to be defined in next section]. Clearly, the parabolic dependence is inadequate for these. For larger widths the difference between $e \delta V$ and $e \delta V_{0}$ increases, the latter retaining a parabolic shape to a good approximation. The difference is due to the contribution of the charge in the 2DEG, which produces a flattening of the bottom of the transverse confining potential. We shall further discuss and clarify this behavior in Sec. VI where we generalize a classical model proposed by Shikin et al. ${ }^{3,4}$

\section{THE TWO-DIMENSIONAL APPROXIMATION}

Repeating an analysis similar to that in Sec. II of Ref. 7, we have found that the wave functions, $\Psi_{l}(x, z)$, determined solving Eq. (20) can be to a good approximation factorized into longitudinal and in plane components. We shall now present an ansatz similar to that introduced for gated wires in that reference and show that it allows us to formulate an accurate factorization approximation. With this ansatz the results found in Sec. II are well reproduced and require a much smaller computational effort. We start by rewriting Eq. (20), as

$$
\begin{aligned}
& {\left[-\frac{\hbar^{2}}{2 m^{*}}\left(\frac{d^{2}}{d x^{2}}+\frac{d^{2}}{d z^{2}}\right)+e \Phi_{0}(0, z)\right.} \\
& \left.\quad+\left[e \Phi(x, z)-e \Phi_{0}(0, z)\right]\right] \Psi_{l}(x, z)=E_{l} \Psi_{l}(x, z),
\end{aligned}
$$

and replace $e \Phi(x, z)-e \Phi_{0}(0, z)$ by a weighted average over $z$ :

$$
U(x) \equiv \int_{z_{2}}^{\infty} A^{2}(z)\left[e \Phi(x, z)-e \Phi_{0}(0, z)\right] d z,
$$

with a normalized weight function, $A(z)$, to be determined in a moment. With this simplification Eq. (41) becomes

$$
\begin{aligned}
& {\left[-\frac{\hbar^{2}}{2 m^{*}}\left(\frac{d^{2}}{d x^{2}}+\frac{d^{2}}{d z^{2}}\right)+e \Phi_{0}(0, z)+U(x)\right] \Psi_{l, a}(x, z)} \\
& \quad=E_{l, a} \Psi_{l, a}(x, z),
\end{aligned}
$$

which is separable and leads to

$$
\begin{gathered}
\left(-\frac{\hbar^{2}}{2 m^{*}} \frac{d^{2}}{d z^{2}}+e \Phi_{0}(0, z)\right) A(z)=E_{a}^{(z)} A(z), \\
\left(-\frac{\hbar^{2}}{2 m^{*}} \frac{d^{2}}{d x^{2}}+U(x)\right) \phi_{l}(x)=E_{l, a}^{(x)} \phi_{l}(x),
\end{gathered}
$$

with

$$
\begin{gathered}
\Psi_{l, a}(x, z)=\phi_{l}(x) A(z), \\
E_{l, a}=E_{a}^{(z)}+E_{l, a}^{(x)} .
\end{gathered}
$$

Note that we have chosen the weight function to be precisely the solution of Eq. (44). In the previous section we have stressed that the main difference between $e \Phi(0, z)$ and $e \Phi_{0}(0, z)$ was a roughly constant term, so that the $A(z)$ pre-

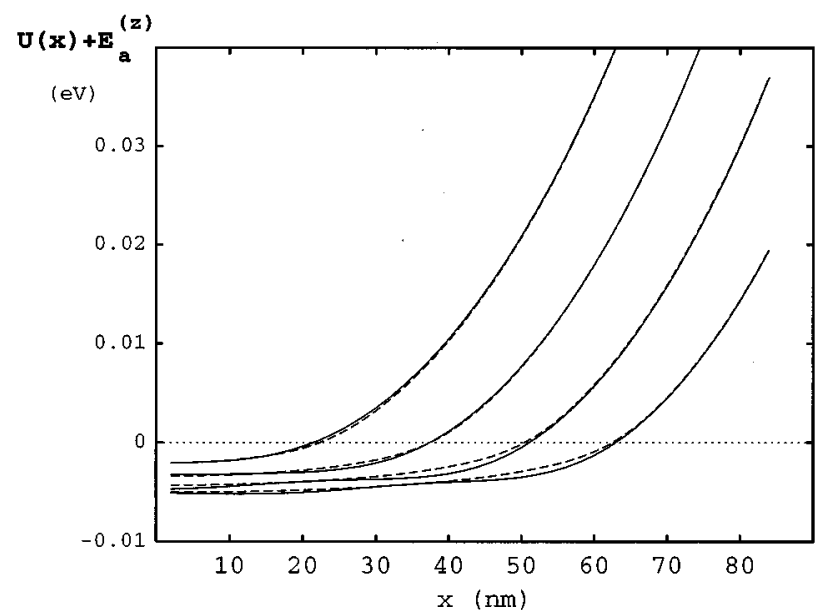

FIG. 7. Effective confining potential $U(x)+E_{a}^{(z)}$ in the 2D approximation (continuous lines) and in Thomas-Fermi (dashed lines).

dicted by (44) can be expected to be very similar to the effective transverse wave function that would be found if, as done in Ref. 7, $e \Phi(0, z)$ had been used in setting up the factorization ansatz. This roughly constant shift enters with opposite signs in the values of $E_{a}^{(z)}$ and $E_{l, a}^{(x)}$ and its effect should almost cancel in their sum, $E_{l, a}$.

In this approach the two-dimensional density is obtained as

$$
\sigma_{2 \mathrm{D}}(x)=\sum_{l} f_{l}\left|\phi_{l}(x)\right|^{2}
$$

where $f_{l}$ has been defined in Eq. (21).

The predictions for the different quantities of interest have been already included in Figs. 2-6. As can be seen in Figs. 2 and 3 the densities are reproduced very accurately, not only the $\lambda(w)$ which is an integrated property, but also the detailed shapes of $\sigma(x)$ for the different $w$, with even the inner oscillations being accurately predicted. In addition, Fig. 4 shows that the longitudinal profile of the density is also very well described. This confirms that the basic assumptions behind this ansatz are correct, and that to a very good approximation (i) the separation of longitudinal and transverse degrees of freedom is justified, and (ii) the longitudinal profile of the electron distribution can be predicted directly from $e \Phi_{0}(0, z)$, i.e., ignoring the roughly constant contribution of the 2DEG, and thus without need for a redetermination of $A(z)$ at each iteration of the Poisson-Schrödinger process. Due to this, with the factorization ansatz one achieves a considerable reduction in the time of computation, because the numerical determination of the $\phi_{l}$ from Eq. (45) is much faster than that of the $\Psi_{l}$ from Eq. (20): a complete Poisson + Schrödinger calculation with this ansatz requires only 2 min in the same 486-33 PC mentioned above.

The transverse confining potential $U(x)$, defined in Eq. (42), is shown in Fig. 7. As remarked in the previous section, the parabolic approximation is only adequate in the limit of vanishing electron gas. The potential due to the charge in the 2DEG flattens out the bottom of the parabola and produces an almost constant inner zone that increases with $w$. 


\section{THOMAS-FERMI APPROXIMATION FOR THE 2DEG}

The accuracy of the factorization ansatz indicates that to a good approximation the electron gas behaves as two dimensional, with an effective confining potential, $U(x)$, determining the electrons wave functions via Eq. (45). At zero temperature then the occupied subbands are those for which the energy, $E_{l, a}$ is lower than the chemical potential, which in our convention is at zero energy. Therefore, Eq. (46) shows that the effective Fermi level for a two-dimensional gas is $E_{F}^{2 \mathrm{D}}=-E_{a}^{(z)}$. The next step is to further simplify our description by using the Thomas-Fermi approximation: guided by Eq. (45), we define the local Fermi momentum in terms of the corresponding $2 \mathrm{D}$ density by the relations

$$
\begin{gathered}
\frac{\hbar^{2}}{2 m^{*}} k_{F}^{2}(x)+U(x)=E_{F}^{2 \mathrm{D}}, \\
k_{F}^{2}(x)=2 \pi \sigma_{\mathrm{TF}}(x) .
\end{gathered}
$$

These allow one to determine the electron density directly from the confining potential $U(x)$ and thus suppress the need to solve the Schrödinger equation (45) for the $\phi_{l}$ 's at each iteration of the self-consistent process.

This Thomas-Fermi method leads to quite accurate estimates for the bulk properties of the electron gas, as shown in Figs. 2 and 3: for the variation of linear density vs $w$ the prediction is practically indistinguishable from that of the $2 \mathrm{D}$ ansatz, whereas for $\sigma(x)$ the prediction is good for the overall size and scale, but the shape does not exhibit the quantal oscillations due to contributions of each subband. The confining potential is shown in Fig. 7. Again the agreement with the $2 \mathrm{D}$ prediction is excellent.

\section{APPROXIMATE ANALYTICAL MODEL}

The above Thomas-Fermi model is based on a semiclassical approximation for the kinetic energy. We find that the resulting charge densities are rather well represented by a simple form proposed by Shikin et al.: $:^{3,4}$

$$
\sigma_{S}(x)=\sigma_{0} \sqrt{1-\left(\frac{x}{R}\right)^{2}} .
$$

Remarkably, this density gives a Hartree potential that is purely quadratic. Suppose, following the analytic results of Sec. III, in particular Eq. (40), that the net confining potential due to all sources other than the charges in the $2 \mathrm{DEG}$ is parabolic and written as

$$
U_{c}(x)=U_{0}+\frac{1}{2} k x^{2}
$$

Then the Thomas-Fermi equation becomes

$$
E_{F}^{2 \mathrm{D}}=U_{0}+\frac{1}{2} k x^{2}+U_{e}(x)+\frac{\hbar^{2} \pi}{m^{*}} \sigma_{\mathrm{TF}}(x),
$$

with

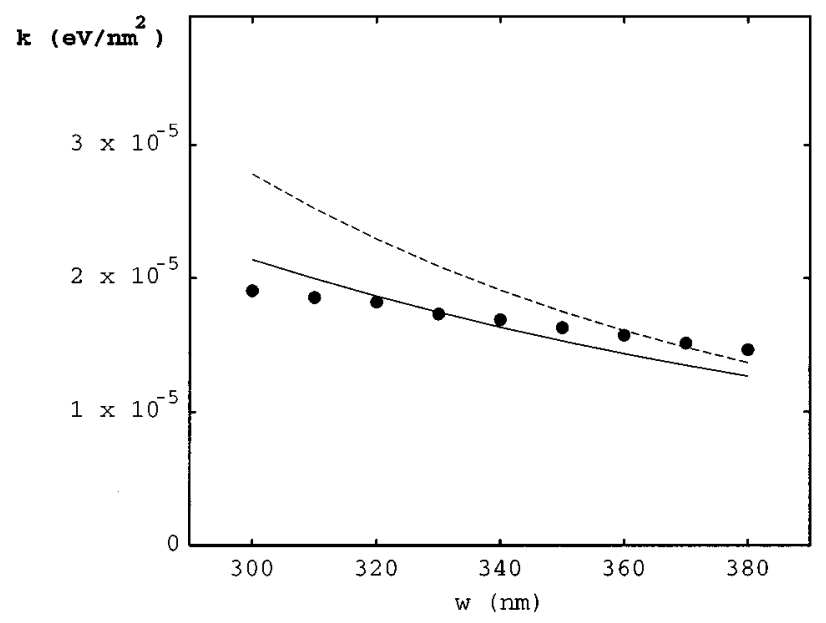

FIG. 8. Dots: effective spring constant $k$ of Eq. (53). Dashed line is from Eq. (40) and the solid line includes the next order correction.

$$
\begin{aligned}
U_{e}(x) & =\frac{-2 e^{2}}{\varepsilon_{r}} \int \ln \frac{|t-x|}{L} \sigma_{S}(t) d t \\
& \simeq \frac{e^{2}}{\varepsilon_{r}} \pi R \sigma_{0}\left[\frac{1}{2}+\ln \left(\frac{2 L}{R}\right)-\frac{x^{2}}{R^{2}}\right], \quad|x| \leqslant R .
\end{aligned}
$$

Here, $L^{2}=D^{2}+R^{2} / 4$, and $D$ is the distance to the mirror charge vertically above the 2DEG. This approximation is developed in Appendix C.

In the classical limit adopted by Shikin et al., one simply neglects the (small) contribution of the kinetic energy which is of order $\hbar^{2}$. Then Eq. (51) is satisfied for $|x| \leqslant R$ with $\sigma_{\mathrm{TF}}(x)=\sigma_{S}(x)$ and

$$
\begin{gathered}
\sigma_{0}=\frac{\varepsilon_{r}}{e^{2}} \frac{k R}{2 \pi}, \\
R^{2}=\frac{4 \mu}{k[1+2 \ln (2 L / R)]}\left[1-\frac{R^{2}}{2 L^{2}}\left(1-\frac{R^{2}}{2 D^{2}}\right)\right],
\end{gathered}
$$

where $\mu=E_{F}^{2 \mathrm{D}}-U_{0}$ is a shifted Fermi level. The linear density, defined as in Eq. (23), becomes

$$
\lambda^{S}=\frac{R \pi}{2} \sigma_{0}
$$

From the numerical Thomas-Fermi densities, $\sigma_{\mathrm{TF}}(x)$, we then read off values of $\sigma_{0}$ and $R$, and use the three equations above to check the validity of this classical model: (i) In all cases considered but one, $\lambda^{\mathrm{TF}}$ is given to within $1 \%$ by the $\lambda^{S}$ of Eq. (55). (ii) From Eq. (53), one deduces an effective value for the oscillator constant $k$. In Fig. 8, this $k$ is compared against values obtained from Eq. (40) and one sees qualitative agreement. (iii) From Eq. (54) one extracts an effective value for the shifted Fermi level $\mu$. Subtracting the corresponding $E_{F}^{2 \mathrm{D}}$ then determines $U_{0}$. We find that $U_{0} \simeq 30 \mathrm{meV}$, independent of the value of $w$ which agrees rather well with the difference between the upper and lower lines in Fig. 5, already discussed just following Eq. (40). From all these comparisons we conclude that the $\sigma_{S}(x)$ in 
Eq. (49) is a very useful model for the density profile of the 2DEG in a deep-etched wire: not only does it explain the flattening of the bottom of the transverse confining potential via very simple analytic expressions, but, in addition, it is reasonably accurate in its main predictions. In particular, due to the long range of the Coulomb force, we find that the Shikin density gives a very good approximation, Eq. (52), for the 2DEG contribution to the electrostatic potential.

\section{CONCLUSION}

We have considered the self-consistent solution of the Poisson and Schrödinger equations for a deep-etched linear quantum wire. An efficient numerical technique has been developed, based on a Fourier expansion of the relevant potentials and densities, which greatly reduces the length of such calculations. Two limiting cases, that of infinitely deep etching, and the wide base approximation, lead to the same approximate analytic forms for the confining potential, and this has allowed us to formulate a simple ansatz for the factorization of the wave functions into vertical and transverse factors that leads to very accurate predictions. In addition, this gives a further improvement in computing times, and justifies the commonly made ab initio assumption that the electron gas is two dimensional.

Working in this two-dimensional approximation, we have implemented a Thomas-Fermi model which is found to give good results for the bulk properties of the 2DEG. Furthermore, the resulting Thomas-Fermi density function has been shown to be very well represented by the analytic form due to Shikin et al. ${ }^{3,4}$ This analytic form leads to useful relations among the central electron density, its radius, the Fermi level, and the effective spring constant of the confining potential, which are accurately obeyed by the numerical Thomas-Fermi results.

\section{ACKNOWLEDGMENTS}

We are grateful to DGICYT, Spain for continued support through Grant Nos. PB91-0236 and PB94-0900 (J.M.). The work of D.W.L.S. was supported by NSERC Canada under research Grant No. OGP00-3198 and by DGICYT.

\section{APPENDIX A: NUMERICAL STRATEGY}

To solve Eq. (17), one has to invert sizable matrices, typically 24 terms in the Fourier series. We have found that it is very accurate and fast to use the following method, based on the analytic knowledge of the approximate solutions, $a_{n}^{(\infty)}$. Defining

$$
\delta a_{n}=a_{n}-\gamma_{n}\left(e^{-\beta_{n} z_{2}}-1\right),
$$

Eq. (17) can be rewritten as

$$
\sum_{m} B_{n, m} \delta a_{m}=F_{n}
$$

with

$$
\begin{aligned}
F_{n}= & \frac{W}{w} \sum_{m} \epsilon_{m, n}\left[\frac{e^{2} \rho_{d} w^{2}}{\varepsilon} \gamma_{m}\left(1-\cosh \beta_{m} z_{2}\right)\right. \\
& \left.\times e^{-\beta_{m} z_{4}}+v_{m}^{(c h)}\left(z_{4}\right)\right]+\frac{e^{2} \rho_{d} w^{2}}{\varepsilon} \beta_{n} \gamma_{n}\left(\cosh \beta_{n} z_{2}-1\right) \\
& \times e^{-\beta_{n} z_{4}}+v_{n}^{\prime}(c h)\left(z_{4}\right) .
\end{aligned}
$$

This has the advantage of suppressing the largest terms that contribute to both sides of Eq. (17) but mutually cancel, so that the smaller contribution due to the $\delta a_{m}$ is enhanced. Likewise one redefines

$$
\begin{aligned}
\widetilde{B}_{n, m} & \equiv B_{n, m} e^{-\beta_{m} z_{4}}, \\
\delta \widetilde{a}_{m} & \equiv \delta a_{m} e^{\beta_{m} z_{4}} .
\end{aligned}
$$

We then solve Eq. (A2) by successive approximations, using as starting values those determined from the diagonal terms of $\widetilde{B}$, i.e., we decompose it into its diagonal and nondiagonal parts:

$$
\widetilde{\mathbf{B}}=\widetilde{\mathbf{B}}^{(D)}+\widetilde{\mathbf{B}}^{(N D)}
$$

and have

$$
\begin{gathered}
\delta \widetilde{a_{0}}=\widetilde{\mathbf{B}}^{(D)-1} \mathbf{F}, \\
\delta \widetilde{a_{i}}=\delta \widetilde{a_{0}}-\widetilde{\mathbf{B}}^{(D)-1} \widetilde{\mathbf{B}^{(N D)}} \delta{\widetilde{a_{i-1}}} .
\end{gathered}
$$

Iterating the latter a few times suffices to get the required accuracy.

\section{APPENDIX B: A TAYLOR EXPANSION} Since

In general, consider a function $f(x)=f(\exp (\varepsilon)) \equiv F(\varepsilon)$.

$$
\frac{d}{d \varepsilon}=x \frac{d}{d x}
$$

the coefficients in the Taylor expansion of $F(\varepsilon)$ require the derivatives

$$
F^{(n)}(\varepsilon)=\left(x \frac{d}{d x}\right)^{n} f(x)
$$

evaluated at $\varepsilon=0$ and $x=1$, respectively. Applying this general result to the function $S(\xi)$, defined in (35), and changing the sign of $\varepsilon$, we have 


$$
\begin{aligned}
S\left(\xi=e^{-\varepsilon}\right)= & S(\xi=1)-\varepsilon S^{\prime}(\xi=1) \\
& +\frac{1}{2} \varepsilon^{2}\left[S^{\prime}(\xi=1)+S^{\prime \prime \prime}(\xi=1)\right] \\
& -\frac{1}{6} \varepsilon^{3}\left[S^{\prime}(\xi=1)+3 S^{\prime \prime}(\xi=1)+S^{\prime \prime \prime}(\xi=1)\right] \\
& +\frac{1}{24} \varepsilon^{4}\left[S^{\prime}(\xi=1)+7 S^{\prime \prime}(\xi=1)+6 S^{\prime \prime \prime}(\xi=1)\right. \\
& \left.+S^{(i v)}(\xi=1)\right]+\cdots .
\end{aligned}
$$

The power series expansion in Eq. (35) is convergent for $\xi$ inside the unit circle, and therefore defines the function $S$ uniquely, but as it stands it can be used to compute derivatives only up to order 2, since the expressions for higher orders become divergent. Therefore we redefine $S(\xi)$ as

$$
S(\xi)=\int_{0}^{\xi} \frac{\xi \xi^{\prime}}{\xi^{\prime}} \int_{0}^{\xi^{\prime}} \frac{\arctan \xi^{\prime \prime}}{\xi^{\prime \prime}} d \xi^{\prime \prime}
$$

which can be easily shown to be equivalent to the previous definition using the series expansion of $\arctan x$. This expression allows one to compute derivatives to all orders. Applying Eq. (B2) to Eq. (B4) gives

$$
\begin{gathered}
S^{\prime}(\xi=1)+S^{\prime \prime}(\xi=1)=\frac{\pi}{4}, \\
S^{\prime}(\xi=1)+3 S^{\prime \prime}(\xi=1)+S^{\prime \prime \prime}(\xi=1)=\frac{1}{2}, \\
S^{\prime}(\xi=1)+7 S^{\prime \prime}(\xi=1)+6 S^{\prime \prime \prime}(\xi=1)+S^{(i v)}(\xi=1)=0, \\
S^{\prime}(\xi=1)+15 S^{\prime \prime}(\xi=1)+25 S^{\prime \prime \prime}(\xi=1)+10 S^{(i v)}(\xi=1)+S^{(v)} \\
\times(\xi=1)=-\frac{1}{2}, \\
S^{\prime}(\xi=1)+31 S^{\prime \prime}(\xi=1)+90 S^{\prime \prime \prime}(\xi=1)+65 S^{(i v)}(\xi=1) \\
+15 S^{(v)}(\xi=1)+S^{(v i)}(\xi=1)=0, \ldots,
\end{gathered}
$$

and one obtains Eq. (36).

\section{APPENDIX C: THE SHIKIN POTENTIAL INCLUDING MIRROR TERMS}

We wish to show that the Shikin density gives a harmonic contribution to the lateral confining potential felt by the electrons. To begin, note that a filament of unit charge per unit length at position $x$ gives a potential

$$
e V_{f}(t, x)=-\frac{2 e^{2}}{\varepsilon_{r}} \ln |t-x|,
$$

so that the Hartree potential due to the charge distribution (49) is

$$
U_{e, H}=\frac{-2 e^{2} \sigma_{0}}{\varepsilon_{r}} \int_{-R}^{R} \sqrt{1-\left(\frac{x}{R}\right)^{2}} \ln |t-x| d x \equiv \frac{-e^{2} \sigma_{0}}{\varepsilon_{r}} G(t) .
$$

The derivative is

$$
\begin{aligned}
\frac{d G(t)}{d t} & \equiv g(t)=2 \int_{-R}^{R} \frac{\sqrt{1-\left(\frac{x}{R}\right)^{2}}}{t-x} d x \\
& =-2 \int_{-1}^{1} \frac{\sqrt{1-x^{\prime 2}}}{x^{\prime}-z} d x^{\prime},
\end{aligned}
$$

with $z=t / R$. This latter integral is given on p. 246 of Ref. 10:

$$
\begin{aligned}
g(t) & =2 \pi z, \quad|t|<R \\
& =2 \pi\left[z-\sqrt{z^{2}-1}\right], \quad|t|>R .
\end{aligned}
$$

This result establishes the quadratic potential inside the charge distribution. Direct evaluation of $G(t=0)$ then leads to Eq. (52).

The mirror charge which we take into account is that due to a layer of charge at distance $D=2 z_{2}$ above the electron gas. Corresponding mirror terms reflected in the sides of the etched wire are neglected because the lateral dimension $w \gg D>R$. Then similarly to Eq. (68),

$$
U_{e, m}(x)=\frac{e^{2} \sigma_{0}}{\varepsilon_{r}} G_{m}(x),
$$

with

$$
G_{m}(x)=\int_{-R}^{R} \sqrt{1-\left(\frac{t}{R}\right)^{2}} \ln \left[(t-x)^{2}+D^{2}\right] d t .
$$

The argument of the logarithm may be expanded as

$$
\ln \left[(t-x)^{2}+D^{2}\right]=\ln \left[D^{2}+x^{2}\right]+\frac{t^{2}-2 x t}{D^{2}+x^{2}} \cdots
$$

and the result of integrating over $t$ can be expressed as

$$
\begin{aligned}
G_{m}(x) & \approx \frac{\pi R}{2} \ln \left[D^{2}+x^{2}+\frac{R^{2}}{4} \frac{D^{2}-x^{2}}{D^{2}+x^{2}}\right] \\
& \approx \pi R\left[\ln L+\frac{x^{2}}{2 L^{2}}\left(1-\frac{R^{2}}{2 D^{2}}\right)+\cdots\right],
\end{aligned}
$$

with a constant $L^{2}=D^{2}+\left(R^{2} / 4\right)$. The first expression is valid to leading order both for small and for large $x^{2}$, and is an excellent interpolation between these limits.

Keeping just the logarithm in the last line of Eq. (C7) is equivalent to saying that the potential due to a filament of charge, Eq. (C1), is modified to read

$$
e V_{f+m}(t, x)=-\frac{2 e^{2}}{\varepsilon_{r}} \ln \frac{|t-x|}{L}
$$

and this leads to Eq. (52). If one also keeps the $x^{2} / L^{2}$ piece of Eq. (C7), then the coefficient of $x^{2}$ in Eq. (52) will be changed. This refinement is already included in Eq. (54). 
We remark that in Ref. 3 there is an undefined quantity $L$ which we can see arises naturally in our treatment as the expression of the boundary condition on the surface of the semiconductor via the mirror term. We also note that in the large $x$ limit, one expands in powers of $1 / x^{2}$. The appropriate $\mathrm{L}, 2=\mathrm{D} 2-(\mathrm{R} 2 / 4)$ from Eq. (C7), and the potential of Eq. (52) reduces correctly to the dipole formed by the filament plus its mirror image.
${ }^{1}$ G.L. Snider, I.-H. Tan, and E.L. Hu, J. Appl. Phys. 68, 2849 (1990).

${ }^{2}$ D. Jovanovic and J.P. Leburton, IEEE Electron Device Lett. 14, 7 (1993)

${ }^{3}$ V. Shikin, T. Demel, and D. Heitmann, Surf. Sci. 229, 276 (1990).

${ }^{4}$ V. Shikin, S. Nazin, D. Heitmann, and T. Demel, Phys. Rev. B 43, 11903 (1991).

${ }^{5}$ R.W. Grant, J.R. Waldrop, S.P. Kowalczyk, and E.A. Kraut, J. Vac. Sci. Technol. 19, 477 (1981).
${ }^{6}$ P. Revva, J.M. Langer, M. Missous, and A.R. Peaker, J. Appl. Phys. 74, 416 (1993).

${ }^{7}$ J. Martorell, Hua Wu, and D.W.L. Sprung, Phys. Rev. B 50, 17298 (1994).

${ }^{8}$ W.H. Press, B.P. Flannery, S.A. Teukolsky, and W.T. Vetterling, Numerical Recipes (Cambridge University Press, Cambridge, England, 1989).

${ }^{9}$ J.H. Davies, Semicond. Sci. Technol. 3, 995 (1988).

${ }^{10}$ Tables of Integral Transforms, edited by A. Erdelyi (McGrawHill, New York, 1954). 